Int. J. Morphol.,

31(1):172-176, 2013.

\title{
Desarrollo de la Médula Espinal de Salmón (Salmo salar) durante el Período Post-eclosional
}

\author{
Development of Spinal Cord of the Salmon \\ (Salmo salar) during the period post eclosional
}

Hilda Hernández* \& Mariana Rojas*;**

HERNÁNDEZ, H. \& ROJAS. M. Desarrollo de la médula espinal de Salmón (Salmo salar) durante el período post-eclosional. Int. J. Morphol., 31(1):172-176, 2013.

RESUMEN: En este estudio se describe el desarrollo post-eclosional de la médula espinal del salmón. Salmo salar. Se utilizaron 200 alevines recién eclosionados, los que fueron cultivados en el Centro de Estudios Acuícolas de la Universidad de Chile. Las condiciones ambientales de cultivo fueron de un $90 \%$ de saturación de oxigeno. La temperatura ambiental se mantuvo en $7^{\circ} \mathrm{C}$. A los días $1,3,5$ 7 ds post-eclosión, 50 alevines por grupo etario fueron anestesiados y sacrificados por exposición a 5\% Benzocaina diluida en agua (Kalmagin 20®, Farquímica). Posteriormente fueron fijados en formalina tamponada al 10\% y procesados mediante técnica histológica. Para cada alevín se tomaron a nivel de la aleta dorsal un total de 40 cortes coronales seriados de $5 \mu \mathrm{m}$ de grosor, los que fueron procesados de acuerdo a las técnicas Cresil violeta. La cuantificación neuronal se realizó sobre imágenes microscópicas mediante el método del disector. Los resultados obtenidos se sometieron a una prueba de Coeficiente de Kurtosis con el propósito de analizar el grado de concentración que presentan los valores alrededor de la zona central de la distribución. La médula espinal de los alevines de 1 día es poco diferenciada. En los alevines de 3, 5 y 7 días se diferencian gradualmente las neuronas de la sustancia gris, pero no presenta la distribución característica en forma de "Y" invertida del salmón adulto. El número de neuronas aumenta desde 67+1.7 en el día 1 hasta 88+2.1 en el día 7. Esta observación se puede relacionar con la ausencia de movimientos natatorios de los peces durante los primeros días ya que estos caen sobre la gravilla al fondo de las bateas. Un factor determinante en la adquisición de la morfología de la médula espinal es el inicio de los movimientos natatorios, lo que ocurre aproximadamente al quinto día post-eclosión. La actividad motriz activa permite que las neuronas de la médula espinal sean reclutadas y se formen y activen las redes neurales, permaneciendo finalmente los circuitos más eficientes. El aumento del número de neuronas se puede explicar por neurogénesis post-eclosión, como ocurre en otros teleósteos. Este estudio indica que al momento de la eclosión, el sistema nervioso está muy indiferenciando, y que durante las primeras semanas de vida del alevín ocurre la diferenciación de las neuronas y neurogénesis. Este conocimiento es muy importante debido a que en las pisciculturas se cuidan las ovas, y se descuida la fase del alevinaje en la creencia que los tejidos están constituidos.

PALABRAS CLAVE: Médula espinal; Sistema nervioso; Neurogénesis; Alevín; Salmón.

\section{INTRODUCCIÓN}

Durante la eclosión, la producción de enzimas proteolíticas y movimientos enérgicos por parte del embrión facilita la ruptura del corion, y la liberación del alevín, el cual mide de 15 a $20 \mathrm{~mm}$. Durante la época en la que tienen adherido el saco vitelino los alevines permanecen en el fondo del tanque. Una vez consumidos los nutrientes del saco vitelino, se elevan y se adhieren al cristal ó a las plantas. (Hernández, 2011). El eje dorsoventral del tubo neural en pez cebra, como en otros vertebrados sigue un patrón tal que diferentes tipos neuronales residen en distintas posiciones. Las neuronas de la cresta neural y neuronas sensoriales surgen del neuroectodermo dorsal, mientras que las neuronas motoras ventrales y varias interneuronas son distribuidas en su mayoría a través de la región intermedia entre las neuronas motoras y neuronas sensoriales (Kuwada et al., 1990; Bernhardt et al., 1990). El establecimiento de los diferentes tipos neuronales a lo largo del eje dorsoventral de la médula espinal es mediada por dos centros de señales: Señales de BMP (proteína morfogenética del hueso) desde dorsal y señales de $\mathrm{SHH}$ (el morfógeno Sonic hedgehog) desde ventral (Nguyen et al., 2000). Aún no se determina cómo los circuitos neuronales se establecen por primera vez desde el comienzo de la 
sinaptogénesis y cómo las redes subyacentes embrionarias se transforman para producir la locomoción. Las contracciones musculares espontáneas aparecen en un momento en el que pocas neuronas han extendido los axones en la médula espinal. Las primeras neuronas proyectadas desde la médula espinal son las motoneuronas que inervan la musculatura ventral, dorsal y media, respectivamente en cada somito

En la médula espinal de salmones adultos, las neuronas están agrupadas en forma de Y invertida en la sustancia gris (González \& Valladolid, 1998). Esta se divide en tres áreas que sirven de entrada o salida a los nervios espinales: las columnas ventral, dorsal y lateral. Por la columna ventral emerge la raíz ventral o motora de los nervios espinales, que inervan los músculos somáticos y los órganos viscerales. Las raíces dorsales sensitivas reciben los estímulos procedentes del organismo a través de los ganglios espinales y penetran en la columna del asta dorsal (Kardong, 2006). Debido a que los alevines no presentan movimientos durante los primeros días después de la eclosión, nos interesó evaluar el estado de desarrollo, de la médula espinal de alevines y cuantificar el número de neuronas entre los días 1-7 post eclosión.

\section{MATERIAL Y MÉTODO}

Se utilizó un total de 250 alevines de salmón Salmo salar en etapa de alevín recién eclosionado, los que fueron cultivados en el Centro de Estudios Acuícolas de la Universidad de Chile, en la ciudad de Castro, por 30 días bajo condiciones de normoxia. Las condiciones ambientales de cultivo fueron de un $90 \%$ de saturación de oxigeno. La temperatura ambiental se mantuvo en $7^{\circ} \mathrm{C}$. A los días $1,3,57$ días post-eclosión, 50 alevines por grupo etario fueron anestesiados y sacrificados por exposición a 5\% Benzocaina diluida en agua (Kalmagin 20®, Farquímica). Posteriormente los alevines fueron anestesiados y fijados en formalina tamponada al $10 \%$, incluidos en parafina, seccionados en un micrótomo (Microm) y montados en porta objetos xilanizados. Para cada alevín se tomaron a nivel de la aleta dorsal un total de 40 cortes coronales seriados de $5 \mu \mathrm{m}$ de grosor, los que fueron procesados de acuerdo a las técnica histoquímicas Cresil violeta.

La descripción de la morfología de la médula espinal de alevines post-eclosión contempló la identificación de una columnas dorsal, y dos ventrolaterales en la sustancia gris de la médula espinal. Los criterios para la identificación de las neuronas presentes en la médula espinal de los alevines recién eclosionados consideraron la morfología neuronal, que incluye núcleo con presencia de nucléolo prominente, límites neuronales definidos, ubicación y forma del cuerpo neuronal. La cuantificación neuronal se realizó sobre imágenes microscópicas mediante el método del disector que consiste en la cuantificación del número de células contenidas en cuadrantes denominados disectores ópticos, distribuidos sistemáticamente en una plantilla previamente diseñada. Los datos obtenidos se sometieron a una prueba de Coeficiente de Kurtosis con el propósito de analizar el grado de concentración que presentan los valores alrededor de la zona central de la distribución.

\section{RESULTADOS}

Los alevines de un día post- eclosión presentaron tejidos poco diferenciados. (Fig. 1). Los arcos neurales que protegen la médula espinal se encuentran en fase mesenquimática. Las neuronas se identifican claramente y es posible diferenciar la sustancia gris de la sustancia blanca. En la región dorsal no se observa cresta neural ni tampoco ganglios nerviosos. Las células progenitoras de neuronas se ubica predominantemente alrededor del canal central que es longitudinal y se extiende desde la región dorsal a la ventral, pero no es posible diferenciar claramente entre las capas del dominio germinativo y del dominio post-mitótico, (Fig. 2). En la región ventral no se distingue la placa del piso. La notocorda que es una estructura hidrodinámica cambia de forma (distendida o contraída), con las consiguentes cambios en la región ventral de la médula espinal.

En todos los alevínes de 3 días post- eclosión se encontraron neuronas gigantes ubicadas dorsalmente en la sustancia gris. Más ventralmente se reconocen células que han aumentado de tamaño, cambiado de forma y que han migrado ventrolateralmente. (Fig. 3).

En los alevines de 5 y 7 días post-eclosión se observa avances en la histogénesis, los arcos neurales se encuentran en fase condrogénica (Fig, 4), El canal central de la médula espinal está más cerrado. La sustancia gris tiene una forma triangular, las neuronas de la región ventral han aumentado de tamaño y han cambiaron su forma de cubica a poligonal, además se comienzan a distribuir ocupando las regiones ventro-laterales de la sustancia gris, diferenciando así las neuronas motoras e interneuronas. En ninguno de los alevines estudiados se observó la forma de "Y" invertida típica del adulto (Figs. 4 y 5).

Al comparar la médula espinal de alevines de un día post eclosión con las de 5 y 7 días, se observó un aumento significativo en el número de neuronas. En la tabla 1 se resume el número de neuronas cuantificadas a los días 1, 3, 5, 7. 


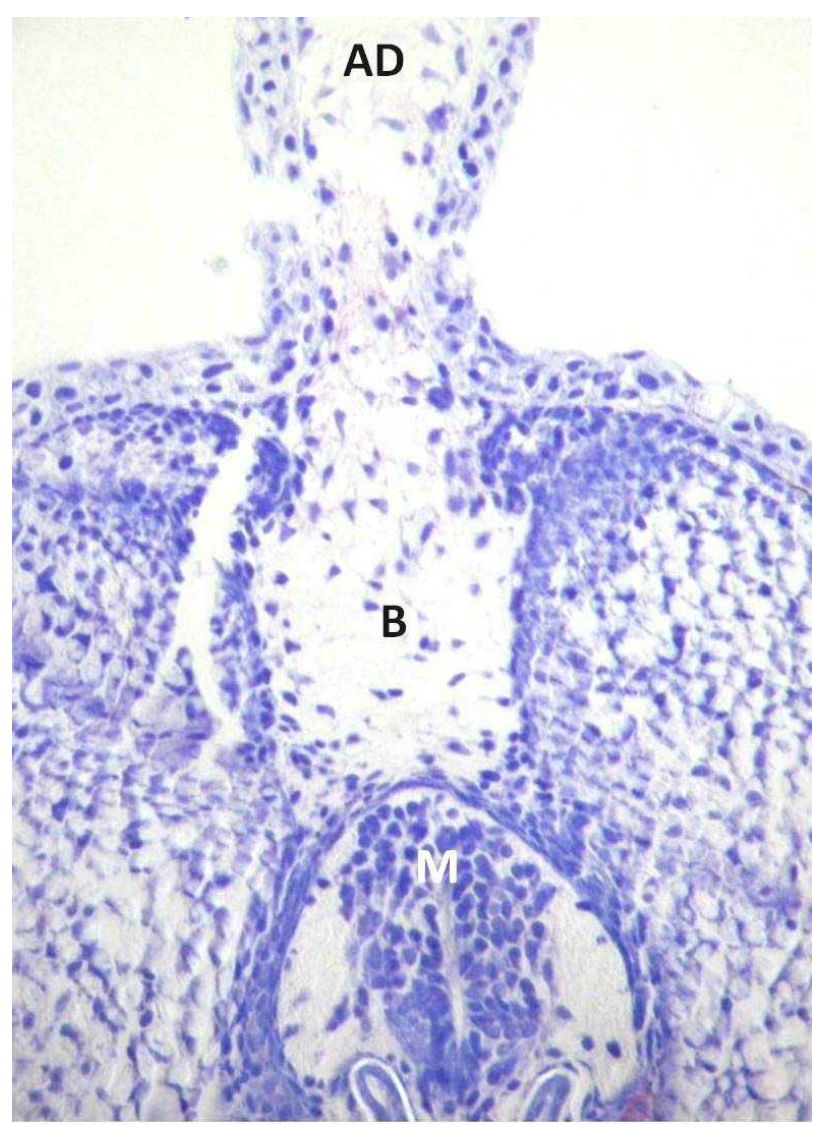

Fig.1 Corte transversal de alevín de 1 día post eclosión. Se observa la aleta dorsal (AD). Médula espinal (M). Blastema de tejido mesenquimal muy laxo (B). Tinción: cresil violeta, 50X

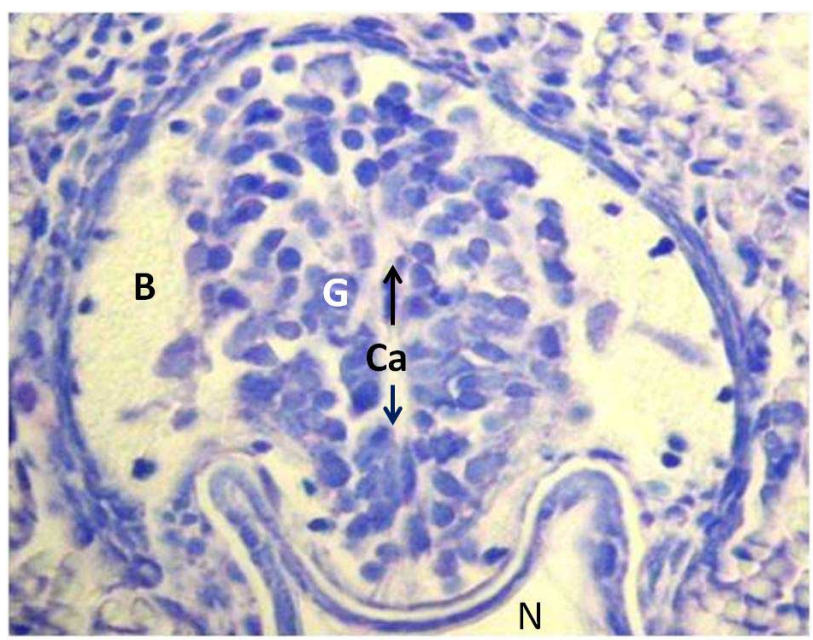

Fig. 2 Corte transversal de médula espinal a nivel de la aleta dorsal de un alevín de un día post-eclosión, El canal central (Ca) es longitudinal y se extiende desde dorsal a ventral. Se observa la distribución neuronal alrededor del canal central, además se identifican neuronas redondas, cromatínicas o alargadas formando la sustancia gris (G) y periféricamente la sustancia blanca (B). Notocorda (N). Tinción: cresil violeta. 400X.

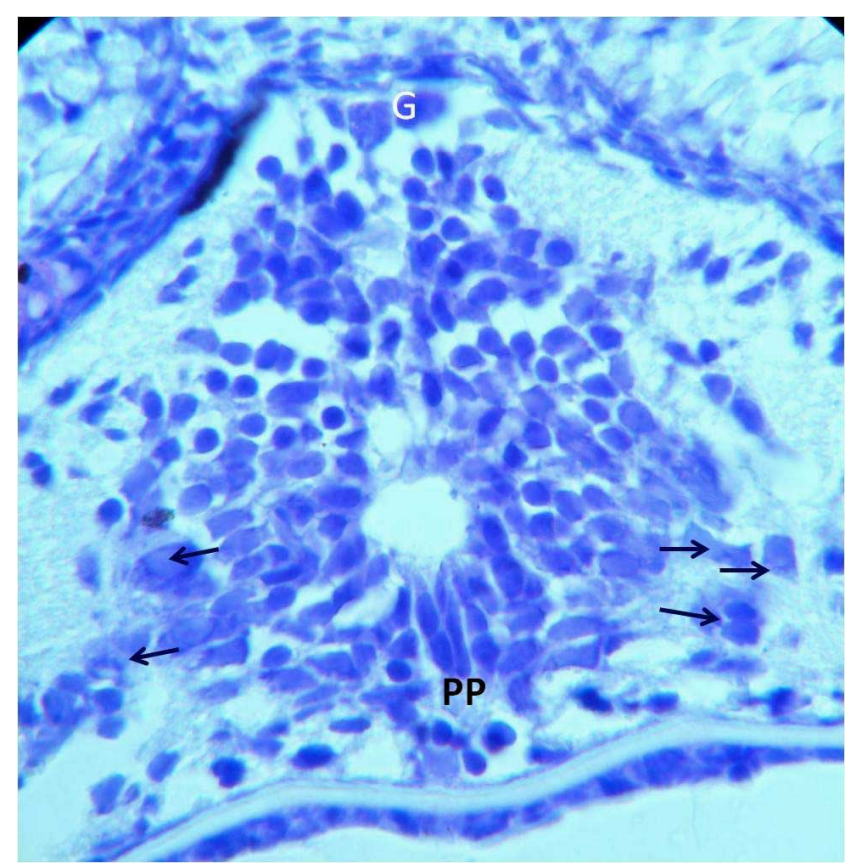

Fig. 3. Corte transversal de médula espinal a nivel de la aleta dorsal de un alevín de tres días post-eclosión. En la región dorsal de la sustancia gris, se encuentran neuronas gigantes $(\mathrm{G})$, ventralmente en la sustancia gris se han diferenciado células que han aumentado de tamaño, cambiado de forma y han migrado ventrolateralmente (flechas). Se está constituyendo la placa del piso (PP). No se puede diferenciar entre el dominio de células progenitoras y el de células postmitóticas. Tinción: cresil violeta 400X.

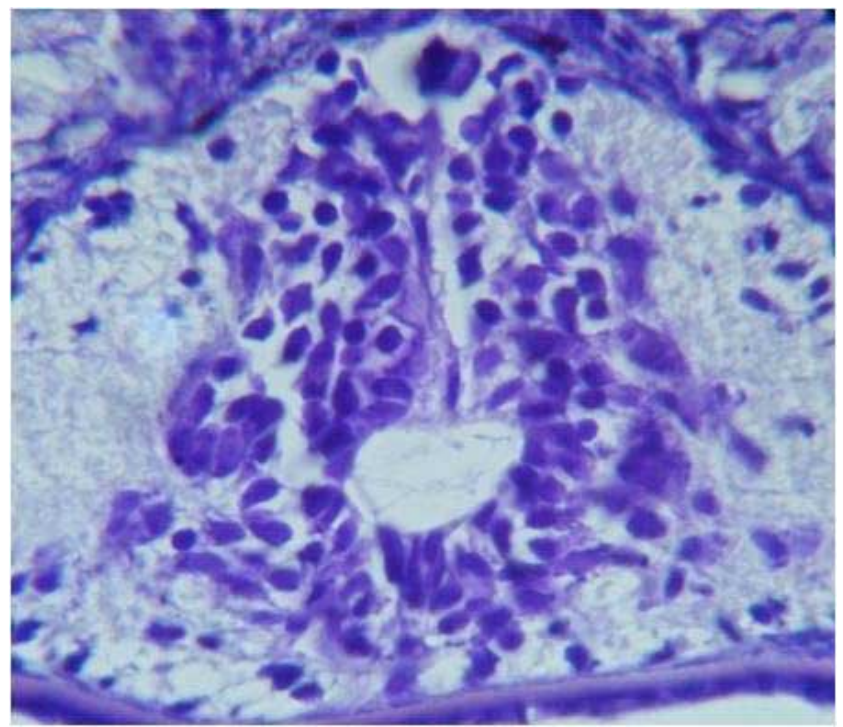

Fig 4. Corte transversal de médula espinal a nivel de la aleta dorsal de un alevín de cinco días post-eclosión. El canal central está más cerrado. Aún no se puede diferenciar el domino de células progenitoras de las células post-mitóticas. Tinción: cresil violeta. 400X. 


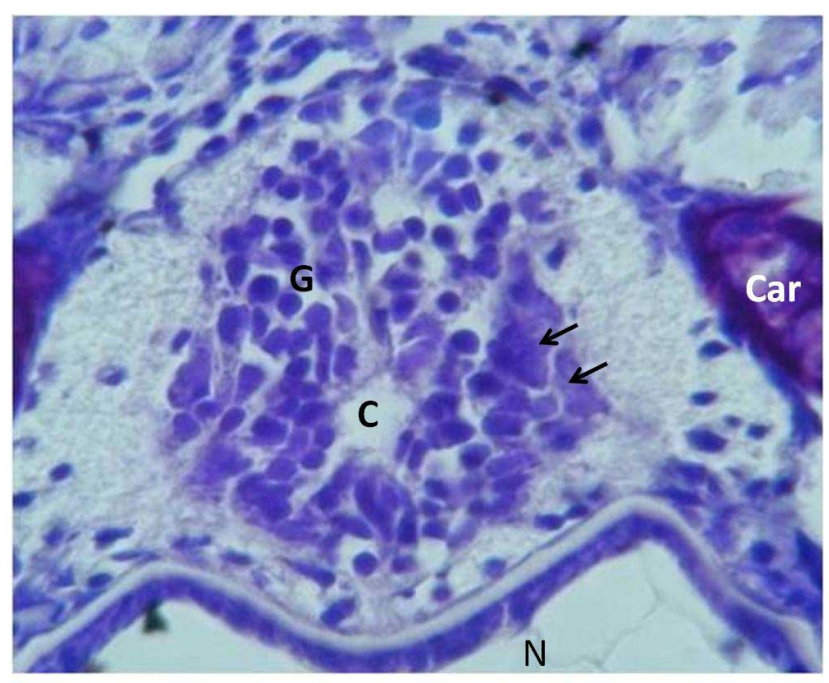

Fig. 5. Corte transversal de médula espinal a nivel de la aleta dorsal de un alevín de siete días post-eclosión. La sustancia gris $(\mathrm{G})$ tiene una forma triangular posiblemente porque la notocorda $(\mathrm{N})$ no está distendida. La sustancia gris $(\mathrm{G})$ presenta neuronas de distintos tamaños. Las neuronas no solo se ubican alrededor del canal central si no que lateralmente se están diferenciando como neuronas motoras. (flechas) e interneuronas. Periféricamente a la médula espinal se observa la formación del cartìlago hialino del arco neural (Car). Tinción: cresil violeta. 1000X.

Tabla 1. Número de neuronas (Media, DS y Varianza) presentes en los cortes seriados de médula espinal de alevín a los $1,3,5$ y 7 ds post-eclosión mantenidos a $7^{\mathrm{a}}$ de temperatura.

\begin{tabular}{lccc}
\hline Día & Media & DS & Varianza \\
\hline 1 & 67,0 & 1,7 & 3,0 \\
3 & 79,5 & 2,5 & 3,5 \\
5 & 84,1 & 2,0 & 3,7 \\
7 & 88,2 & 2,1 & 3,8 \\
\hline
\end{tabular}

\section{DISCUSIÓN}

La médula espinal de los alevines de 1 y 3 días es poco diferenciada, aunque es posible diferenciar entre sustancia blanca y sustancia gris, es difícil reconocer entre las capas de dominio germinativo, y post-mitótica. Esta observación se puede relacionar con la ausencia de movimientos natatorios de los peces durante los días 1 y 3 ya que estos caen sobre la gravilla al fondo de las bateas (Hernández, 2011). En los alevines de 5 y 7 días ocurren pocos cambios, se empiezan a formar gradualmente la columna dorsal y las dos columnas ventrales de la médula espinal. Un factor determinante en la adquisición de la morfología de la médula espinal es el inicio de los movimientos natatorios, lo que ocurre aproximadamente al quinto día post-eclosión. La actividad motriz activa permite que las neuronas de la médula espinal sean reclutadas y se formen y activen las redes neurales, permaneciendo finalmente los circuitos más eficientes Este proceso también ha sido descrito en pez cebra (McLeand \& Fetcho, 2009; Fetcho \& McLean, 2010).

El número de neuronas aumenta desde $67+1.7$ en el día 1 hasta $88+2.1$ en el día 7. El aumento del número de neuronas se puede explicar por neurogénesis post-eclosión, como ocurre en otros teleósteos (Birse et al., 1980; Zupanc, 2001). Este estudio indica que al momento de la eclosión, el sistema nervioso está muy indiferenciando, y que durante las primeras semanas de vida del alevín ocurre la diferenciación de las neuronas y neurogénesis.

La morfología medular en forma de "Y" invertida, característica de la médula espinal en salmones maduros (González \& Valladolid), se adquiere posteriormente en la vida postnatal, cuando ha involucionado el saco vitelino, y el alevín tiene actividad motriz activa (Hernández).

Este estudio indica que al momento de la eclosión, el sistema nervioso está muy indiferenciando, y que durante las primeras semanas de vida del alevín ocurre la diferenciación de las neuronas y neurogénesis, Este conocimiento es muy importante debido a que en las pisciculturas se dan los mayores cuidados a la ova, y se descuida la fase del alevinaje posteclosión en la creencia errónea que sus tejidos ya están completamente constituídos.

HERNÁNDEZ, H. \& ROJAS. M. Development of spinal cord of the salmon. (Salmo salar).during the post eclosional period. Int. J. Morphol., 31(1):172-176, 2013.

SUMMARY: We describe the development of the spinal cord during the post eclosion period of the salmon (Salmo salar). We used a total of 200 newly hatched fry grown in the Aquaculture Research Center of the Universidad de Chile. Environmental conditions were of $90 \%$ oxygen saturation. Ambient temperature was maintained at $7^{\circ} \mathrm{C}$. At days, $1,3,5$ and 7 , posthatching, 50 fry were anesthetized and sacrificed by exposure to $5 \%$ benzocaine diluted in water, (Kalmagin 20 (, Farquímica). They were then fixed in $10 \%$ buffered formalin and processed by histological technique. For each juvenile a total of 40 serial coronal sections of $5 \mu \mathrm{m}$ were taken at the level of the dorsal fin, which were then processed according to cresyl violet techniques. Neuronal quantitation was performed on microscopic images by dissector method. The results obtained were subjected to coefficient Kurtosis test in order to analyze the degree of concentration of values around the central distribution area.The spinal cord of the one-day fry is poorly differentiated. In fry of 3,5 and 7 days neurons are gradually differentiated, they do not however present the characteristic neuronal distribution inverted "Y" of the adult salmon. The number 
of neurons increases from $67 \pm 1.7$ on day one, to $88 \pm 2.1$ on day 7. This observation may be related to the absence of fish swimming movements during days one and three as these fall on the gravel at the bottom of the trays. A determining factor in the acquisition of the morphology of the spinal cord is the start of swimming movements, which occur at around the fifth day posthatching. Active motor activity allows spinal cord neurons to be recruited and form to activate neural networks, to remain finally in the most efficient circuits. Increasing the number of neurons can be explained by post-hatching neurogenesis as in other teleosts. This study indicates that at the time of hatching, the nervous system is very undifferentiated and that neuron differentiation and neurogenesis occur during the first weeks of life. This knowledge is very important as fish farms take care of eggs, neglecting the nursery stage in the belief that tissues are formed.

KEY WORDS: Spinal cord, nervous system, neurogenesis; Alevín; Salmon.

\section{REFERENCIAS BIBLIOGRÁFICAS}

Birse, S.; Leonard, R.; Coggeshall. R. Neuronal increase in various areas of the nervous system of the guppy, Lebistes. J. Comp. Neurol., 194:291-301,1980.

Bernhardt, R. R.; Chitnis, A. B.; Lindamer, L. \& Kuwada, J.Y. Identification of spinal neurons in the embryonic and larval zebrafish. J. Comp. Neurol., 302:603-16, 1990.

Fetcho, J. R. \& Mclean, D. L. Some principles of organization of spinal neurons underlying locomotion in zebrafish and their implications. Ann. N. Y. Acad. Sci., 1198:94-104, 2010.

González, G. \& Valladolid, M. Aspectos Biológicos, anatomía microscópica y enfermedades infecciosas de los salmónidos. Madrid, España, Graficas Summa. 1998. Cap. 11.

Hernández, H. Efecto de la hipoxia en el desarrollo de la médula espinal en salmón (Salmo salar). Tesis para obtener el grado de Magister en Ciencias Biológicas. Facultad de Medicina, Universidad de Chile, Santiago, Chile, 2011.

Kardong, K. Vertebrados: Anatomía Comparada, función y evolución. McGraw-Hill-Interamericana. 2006. p.639.

Kuwada, J. Y.; Bernhardt, R. R. \& Nguyen, N. Development of spinal neurons and tracts in the zebrafish embryo. J. Comp. Neurol., 302:617-28. 1990.

Mcleand, D. L. \& Fetcho, J. R. Spinal interneurons differentiate sequentially from those driving the fastest swimming movements in larval zebrafish to those driving the slowest ones. J. Neurocsi., 29:13566-77, 2009.
Nguyen, V. H.; Trout, J.; Connors, S. A.; Andermann, P.; Weinberg, E. \& Mullins, M. C. Dorsal and intermediate neuronal cell types of the spinal cord are established by BMP signaling pathway. Development, 127:1209-20, 2000.

Zupanc GKH, Adult neurogenesis and neuronal regeneration in the central nervous system of teleost fish. Brain Behav. Evol., 58:250-275, 2001.

\author{
Dirección para correspondencia: \\ Dra. Mariana Rojas R. \\ Laboratorio de Embriología Comparada \\ Programa de Anatomía y Biología del Desarrollo \\ Facultad de Medicina, ICBM, \\ Universidad de Chile \\ CHILE
}

Email: dramrojas@hotmail.com

Recibido : 19-09-2012

Aceptado: 21-11-2012 